\title{
Vibration of Container Ship
}

\author{
Kazuo Umezaki*, Member Kenji Miyamoto*, \\ Katsuo Ohtaka**, Member Koji Kagawa**, Member
}

\begin{abstract}
Summary
The vibration of a container ship has been studied with a chief attention to the horizontal and the torsional vibrations. Some checks on the problems of structural behaviour and of the added virtual mass of water have been made. Centre of virtual mass of water is calculated for the Lewis form sections. The model experiment has been carried out to check the behaviour of coupled vibration. At the last the experiments in a full scale container ship have been made, using the vibration generator and in the sea trials. It is found that the problem is pretty complicated and many investigations are necessary in order to clarify the mechanism of coupled vibration and the rigidities in the torsional vibration. Horizontal vibration, though it is complicated due to the coupling with torsional vibration, is more accessible. The characteristics of the vertical vibration of the container ship are qualitatively the same as those of conventional types of ships. The bottom vibration is significant in the vertical vibration of higher modes which is likely to reduce the natural frequency of the vertical vibration.
\end{abstract}

\section{Introduction}

Recently container ships play an important role in the modern transportation system. The structural behaviour, however, has not been clarified enough because of their short history since appearance. vibration characteristics being not the exception.

On designing the first container ship built in Japan, some investigations have been made on its vibratory characteristics and the results are presented in this report.

As the construction of the container ship is characterized by the large hatch openings, the vibratory behaviour is also effected by the openings. The large openings inevitably reduce the rigidities such as bending, torsional and shear rigidities, of which the torsional rigidity is the most affected. Another effect of the openings is the shift of the centre of shear. As is well known in the strength of materials, the shear centre of the open cross section is substantially different from that of the closed section. These two major effects are concerned with the torsional and the horizontal hull vibration. In view of this, the torsional-horizontal vibration is mainly treated, though the experiméntal data and the relevant discussion are given on the vertical vibration.

As the subject is so complicated that many problems remain unclarified, which should be investigated in the future.

\section{Special characteristics on the horizontal and torsional vibration}

\subsection{Mechanism of coupled vibration.}

In the previous paper ${ }^{2}$ one of the authors studied the coupled vibration of ships taking into account the bending rigidity, the shear rigidity and Saint-Venant rigidity, and it is concluded that the coupling mechanism of the torsional-horizontal vibration of the uniform beam can be approximated by the simple

* Kobe Shipyard and Engine Works, Mitsubishi Heavy Industries, Ltd.

** Nagasaki Technical Institute, Technical Headquarters, Mitsubishi Heavy Industries, Ltd. 
model shown in Fig. 1. Then the coupled frequency can be estimated by the following formula.

$$
N^{2}=\frac{1}{2\left(1-\sigma^{2}\right)}\left\{N_{h}^{2}+N_{t}^{2} \pm \sqrt{\left(N_{h}^{2}+N_{t}^{2}\right)^{2}-4\left(1-\sigma^{2}\right) N_{h}^{2} N_{t}^{2}}\right\}
$$

where $N$; coupled frequency

$N_{h}$; uncoupled horizontal frequency

$N_{t}$; uncoupled torsional frequency

$\sigma$; non-dimensional parameter (see Fig. 1)

On the other hand it has been shown by Matsuura that the analytical solution taking into account the bending rigidity and bending-torsional rigidity agrees with equation (1). Prof. Yamakoshi hase shown ${ }^{4}$ that the analytical solution with the shear rigidity and Saint-Venant torsional rigidity also agrees

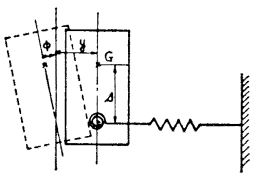

$\sigma=\frac{S}{K} \quad$ s: distance between center of spring $K=\sqrt{\frac{10+W S^{2}}{w}} \quad K:$ rodius of gyration obout center

Fig. 1 Model of mass spring system. with equation (1) Thus it is concluded that mechanism of the coupled torsional-horizontal vibration are hardly changed regardless of the characteristics of the uncoupled vibration. To examine the mechanism in more detail, further considerations are carried out in which the bending rigidity and the Saint-Venant torsional rigidity are taken into account. Equations of motion of the coupled vibration are as follows, neglecting the rotatory inertia:

$$
\left.\begin{array}{l}
\frac{d^{2}}{d x^{2}}\left(p \frac{d^{2} y}{d x^{2}}\right)-a \omega^{2} y=a s \omega^{2} \phi \\
\frac{d}{d x}\left(r \frac{d \phi}{d x}\right)+\left(c+a s^{2}\right) \omega^{2} \phi=-a s \omega^{2} y
\end{array}\right\}
$$

where $\quad y$; horizontal translation of shear center

$\phi$; rotational angle of cross section

$a=w / g$; mass per unit length of beam

$c=I_{G} / g$; mass polar moment of inertia about center of gravity

$p=E I$; bending rigidity of beam

$r=G J=$ Saint-Venant torsional rigidity

$\omega=$ circular frequency

$s=$ distance between shear center and center of gravity

when $y_{n}(x)$ and $\phi_{n}(x)$ are normal modes of the uncoupled horizontal and torsional vibration respectively and $\omega_{b n}$ and $\omega_{t n}$ are their circular frequencies, these satisfy the following equations.

$$
\left.\begin{array}{l}
\frac{d^{2}}{d x^{2}}\left(p-\frac{d^{2} y_{n}}{d x^{2}}\right)=a \omega^{2}{ }_{b n} y_{n} \\
\frac{d}{d x}\left(r \frac{d \phi_{n}}{d x}\right)=-\left(c+a s^{2}\right) \omega_{t n}^{2} \phi_{n}
\end{array}\right\}
$$

The following four terms in the equation of motion are expanded in the series of the normal modes.

$$
\begin{aligned}
y & =\sum_{n} a_{n} y_{n}(x) \\
a s \phi & =\sum_{n} a b_{n} y_{n}(x) \\
\phi & =\sum_{n} c_{n} \phi_{n}(x) \\
a s y & =\sum_{n}\left(c+a s^{2}\right) a_{n} \phi_{n}(x)
\end{aligned}
$$

By the substitution of the equations (3) and (4) to the equation (2), the simultaneous equation as to $a_{n}$ are drawn.

$$
\left.a_{n}=\frac{\omega^{2}}{\omega_{b n^{2}-\omega^{2}}} \cdot \frac{1}{M_{b n}} \sum_{m}\left\{a_{m} \omega^{2} \sum_{k} \frac{1}{\omega_{t k^{2}-\omega^{2}}} \cdot \frac{N_{n k} N_{m k}}{M_{t k}}\right\}\right\}
$$


where,

$$
\left.\begin{array}{ll}
M_{b n}=\int_{0}^{l} a y_{n}^{2} d x & M_{t n}=\int_{0}^{l}\left(c+a s^{2}\right) \phi_{n}^{2} d x \\
N_{i j}=\int_{0}^{l} a s y_{i} \phi_{j} d x &
\end{array}\right\}
$$

The equation (5) is reduced to :

where,

$$
\left(\alpha_{i j}\right)\left(\begin{array}{c}
a_{1} \\
a_{2} \\
\vdots \\
a_{\infty}
\end{array}\right)=\left(\begin{array}{c}
0 \\
0 \\
\vdots \\
0
\end{array}\right)
$$

$$
\begin{aligned}
& \alpha_{i j}=\sum_{k} \frac{\omega^{2}}{\omega_{t k}^{2}-\omega^{2}} \cdot \frac{N_{i k} N_{j k}}{M_{t k}} \quad(i \neq j) \\
& \alpha_{i i}=\sum_{k}\left(\frac{\omega^{2}}{\omega_{t k}{ }^{2}-\omega^{2}} \cdot \frac{N_{i k}{ }^{2}}{M_{t k}}\right)-\frac{\omega_{b i}^{2}-\omega^{2}}{\omega^{2}} M_{b i}
\end{aligned}
$$

The following equation is the secular equation.

$$
\operatorname{det}\left|\alpha_{i j}\right|=0
$$

If $N_{i j}(i \neq j)$ equal zero, $\alpha_{i j}(i \neq j)$ vanishes and secular equation is reduced to :

$$
\alpha_{i i}=0 \quad(i=1,2,3 \cdots \cdots \infty)
$$

Then,

$$
\left(1-\frac{M_{i i^{2}}}{M_{b i} M_{t i}}\right) \omega^{4}-\left(\omega_{b i}^{2}+\omega_{t i}^{2}\right) \omega^{2}+\omega_{b i}^{2} \omega_{t i}^{2}=0
$$

Together with the another assumption that $\sigma$ is constant along the length of the beam, the coupled frequency is expressed as follows :

$$
\begin{gathered}
\omega^{2}=\frac{1}{2\left(1-\chi_{i} \sigma^{2}\right)}\left\{\omega_{b i}{ }^{2}+\omega_{t i}{ }^{2} \pm \sqrt{\left(\omega_{b i}{ }^{2}+\omega_{t i}{ }^{2}\right)^{2}-4\left(1-\chi_{i} \sigma^{2}\right) \omega_{b i}{ }^{2} \omega_{t i}{ }^{2}}\right\} \\
(i=1,2,3 \cdots \cdots \infty)
\end{gathered}
$$

where,

$$
\chi_{i}=\frac{1}{\sigma^{2}} \cdot \frac{N_{i i^{2}}}{M_{b i} M_{t i}}
$$

\begin{tabular}{|c|c|c|c|c|c|c|c|}
\hline$i{ }^{j}$ & 1 & 2 & 3 & 4 & 5 & 6 & 7 \\
\hline 1 & 1.000 & 0 & 0 & 0 & 0 & 0 & 0 \\
\hline 2 & 0 & 0.40529 & 0 & 0.04503 & 0 & 0.01621 & 0 \\
\hline 3 & 0 & 0 & 0.34683 & 0 & 0.06006 & 0 & 0.02626 \\
\hline 4 & 0 & -0.04186 & 0 & 0.34166 & 0 & 0.06795 & 0 \\
\hline 5 & 0 & -0.00007 & -0.06654 & -0.00005 & 0.33649 & -0.00003 & 0.06998 \\
\hline 6 & 0 & -0.00569 & -0.00116 & -0.07739 & -0.00078 & 0.33383 & -0.00050 \\
\hline 7 & 0 & -0.01401 & -0.00280 & -0.01115 & -0.07554 & -0.00793 & 0.33751 \\
\hline
\end{tabular}

The equation (11) coincides with the equation (1) when $\chi_{i}$ equals 1.0 . For the uniform beam, $N_{i j}$ is as follows :

$$
N_{i j}=a s l \int_{0}^{l} y_{i} \phi_{j} d \xi
$$

and their values are shown in Table 1 . From the table we see that their values are small except the diagonal element and that the coupled frequency approximately satisfies the equation (11). The values of $\chi_{i}$ are shown in Table 2. As another example a variable section beam has been studied and the values of $\chi_{i}$ are also shown in Table 2 . The variable section beam in Table 2 has the distribution

Table $1 \int_{0}^{l} y_{i} \phi_{j} d \xi$ of uniform beam 
Table 2 Values of $\chi_{i}$

\begin{tabular}{l|c|c|c}
\hline \hline$i$ & $\begin{array}{c}\text { No. of } \\
\text { nodes }\end{array}$ & $\begin{array}{c}\text { uniform } \\
\text { beam }\end{array}$ & $\begin{array}{c}\text { variable } \\
\text { sect on } \\
\text { beam }\end{array}$ \\
\hline 1 & 0 & 1.0 & 1.0 \\
2 & 1 & 0.98556 & 0.98981 \\
3 & 2 & 0.96233 & 0.97819 \\
4 & 3 & 0.93386 & 0.95169 \\
5 & 4 & 0.90581 & 0.92337 \\
6 & 5 & 0.88773 & 0.91076 \\
7 & 6 & 0.88047 & 0.90600 \\
\hline
\end{tabular}

of the weight in the form of $\sin \pi \xi$. In the calculation of $\chi_{i}$ the normal modes of the uniform beam are used. It is concluded that in these cases the equation (11) (or (1)) is approximately satisfied. The amount of shifting of the frequency due to the coupling effect is larger in the higher frequency mode than in the corresponding lower frequency mode, which is shown by the following procedure. The equation (10) is reduced to :

$$
\left(\omega^{2}-\omega_{b i}{ }^{2}\right)\left(\omega^{2}-\omega_{t i}{ }^{2}\right)=\chi_{i} \sigma^{2} \omega^{4}
$$

The coupled frequencies are the cross points of the both sides of (12). Drawing figures of the right and left hand

\subsection{Structural properties}

\section{TORSIONAL RIGIDITY}

It is necessary to consider the warping rigidity if the ship structure has large and wide hatch openings since the torsional rigidity calculated by Saint-Venant theory decreases in such structures. It is however complicated and difficult to consider and evaluate the warping rigidity of the actual ship structure, and the mechanism of the coupled vibration is subjected to small influence regardless of the nature of the rigidities as mentioned above. So the effect of the warping on the uncoupled torsional vibration only is studied. After additional calculations to the results obtained by Matsuura ${ }^{(3)}$, the torsional natural frequency is expressed as follows :

where,

$$
f=\frac{C_{n}}{2 l} \sqrt{\frac{G J}{I_{p}}}
$$

$$
\begin{aligned}
C_{n} & =\frac{\Omega}{\pi \sqrt{\lambda}} \\
\lambda & =G J \cdot l^{2} / E \Gamma \\
G J & =\text { Saint-Venant torsional rigidity } \\
E \Gamma & =\text { bending-torsional rigidity } \\
l & =\text { length of beam } \\
\Omega^{2} & =I_{p} \omega^{2} l^{1} / E \Gamma \\
I_{p} & =\text { mass polar moment of inertia per unit length of beam } \\
\omega & =\text { circular frequency }
\end{aligned}
$$

The relation between $C_{n}$ and the number of nodes are shown in Fig. 2 corresponding to two end conditions, warping free and prevented. From the figure we see the followings.

(a) The warping rigidity raises the natural frequency. The amount of the effect increases as the number of nodes increases.

(b) The difference of the frequency is not so much between two end conditions.

On the other hand, the influence of the shear deflection on the warping rigidity has been also pointed out by Matsuura and he introduced the shear-torsional rigidity ${ }^{3)}$. The relation between the eigenvalue and the number of nodes are shown in Fig. 3, provided that the bending-torsional rigidity $(E \Gamma)$ and the shear-torsional rigidity $(G S)$ are taken into account and Saint-Venant torsional rigidity is neglected. From the figures we see the followings.

(c) The shear deflection lowers the natural frequency. The amount of the effect increases as the number of nodes increases.

(d) The difference of the frequency between two end conditions decreases as the number of nodes increases. 


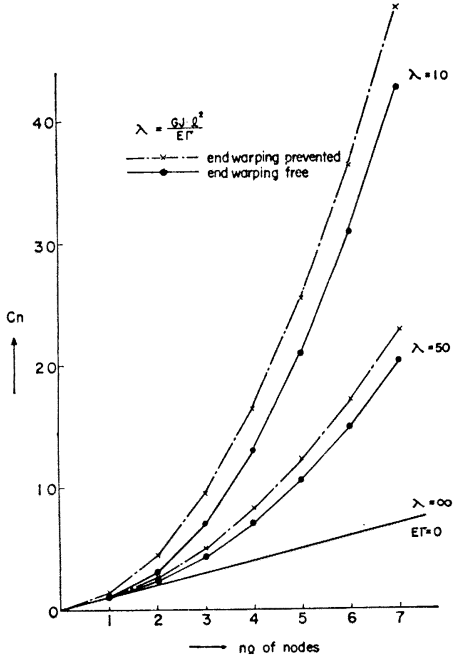

Fig. 2 Relation between number of nodes and $C_{n}$.

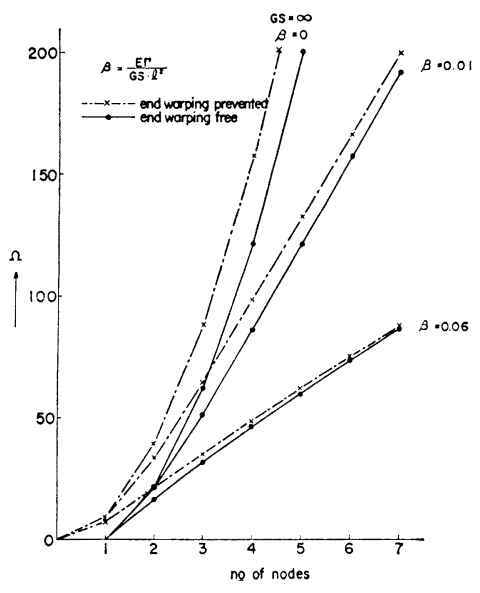

Fig. 3 Relation between number of nodes and $\Omega$.

From the results mentioned above, it is considered that the effect of the bending-torsional rigidity and the shear-torsional rigidity on the natural frequency cancel each other to some extent. The relation between these torsional rigidities can be shown in Fig. 4 from the following procedure.

The eigenvalue of the torsional vibration is expressed as follows, provided that the end warping is prevented.

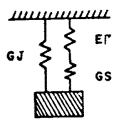

Fig. 4 Relation between three torsional rigidities.

$$
\begin{array}{ll} 
& \Omega=n \pi \sqrt{(n \pi)^{2}+\lambda} \\
\text { or } \quad & \Omega=(n \pi)^{2} / \sqrt{1+(n \pi)^{2} \beta}
\end{array}
$$

When $\Omega_{b}$ and $\Omega_{t}$ are the eigenvalues corresponding to $\lambda=0$ and infinity respectively, following equations are drawn.

$$
\text { and } \quad \begin{array}{cc}
\Omega_{b}=(n \pi)^{2}, & \Omega_{t}=n \pi \sqrt{\lambda} \\
& \Omega^{2}=\Omega_{b}{ }^{2}+\Omega_{t}{ }^{2}
\end{array}
$$

Similary, when $\omega_{b}$ and $\omega_{s}$ are corresponding to $\beta=0$ and infinity respectively, following equations are drawn.

$$
\Omega_{b}=(n \pi)^{2}, \quad \Omega_{s}=n \pi / \sqrt{ } \beta
$$

and

$$
\frac{1}{\Omega^{2}}=\frac{1}{\Omega_{b}^{2}}+\frac{1}{\Omega_{s}^{2}}
$$

The conditions represented by equations (17) and (19) are the same as the case shown in Fig. 4 where a mass is supported by two parallel and series springs respectively.

\section{SHEAR RIGIDITY}

Some definitions are used in respect to the shear rigidity of the beam, and not necessarily agree each other. Web area method is expedient. It is difficult to apply the neutral axis method to the multicellular section. Under the assumption that there is the shear strain over the cross section in proportion to the shear stress and that the work done of the external force is equal to the strain energy of the beam, the energy method gives the following equation.

$$
k^{\prime}=\frac{V^{2}}{A \int_{A} \tau^{2} d A}
$$


where

$$
\begin{aligned}
k^{\prime} & =\text { Shear rigidity coefficient } \\
V & =\text { Shear force } \\
A & =\text { Cross sectional area } \\
\tau & =\text { Shear stress }
\end{aligned}
$$

Applying this method to the box beam and the channel, however, the shear rigidity decreases. A comparison between these three difinitions is shown in Fig. 5. A cause of the decrease of the rigidity defined by the energy method is considered to be that the shear strain on the flange is taken into account. Present method in Fig. 5 is the case where the shear strain on the Web plate only is taken into consideration. Though the true shear rigidity of the actual structure lies between the values of the energy method and present method, it is near the value of present method for the beam with the wide flange plates and short length.

\section{BEHAVIOUR OF CROSS DECK}

Reduced thickness method is adopted as usual in order to consider the effect of the cross deck plate. ${ }^{(8)(13)}$. However, it is necessary to reconsider the effect of the cross deck of ships having large deck openings.

When the ship girder is subjected to the torsion, the shear strain on the deck plate of effective thinckness shown in Fig. 6(a) is expressed as follows :

$$
\gamma_{e}=\frac{X}{G t_{e}}
$$

where, $X=$ shear flow

The shear strain of the cross deck shown in Fig. 6(b) is considered equal to that of the effective plate, the following equation is drawn.

$$
t_{e}=\frac{k^{\prime} A_{d}}{a}\left(1+\frac{b^{2} k^{\prime} G A_{d}}{12 E I_{d}}+\frac{a b k^{\prime} G A_{d}}{24 E I_{s}}\right)^{-1}
$$

The value $k^{\prime} A_{d} / a$ is the reduced thickness and the second and third terms in the bracket show the effect of the bending deflection of the cross deck and the deformation of the ship side structure respectively. The second term is reduced to :

$$
\frac{b^{2} k^{\prime} G A_{d}}{12 E I_{d}}=\frac{k^{\prime} G}{E}\left(\frac{b}{h}\right)^{2} \fallingdotseq \frac{1}{3}\left(\frac{b}{h}\right)^{2}
$$

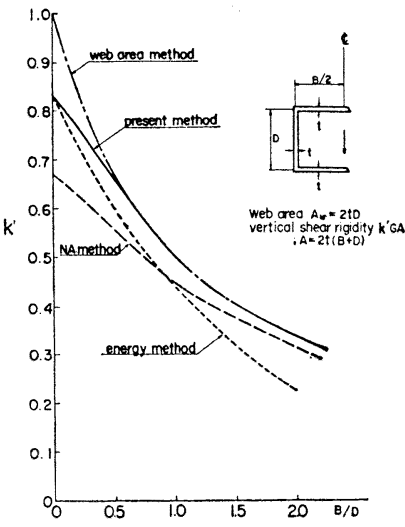

Fig. 5 Coefficient of shear rigidity.

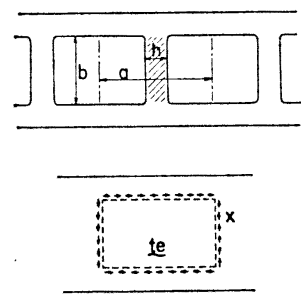

(a)

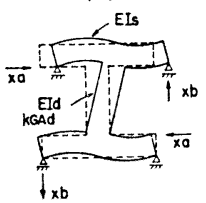

(b)

Fig. 6 Behaviour of cross deck and effective thickness.

Though the value of $b / h$ in the ordinary cargo ship is about 1.0 , it is neary 4.0 in the container ship and reduced thickness method may lead to the overestimation.

\section{CENTER OF TORSION}

In the ordinary type ship, its shear center is near the center of section and the center of torsion is considered to coincide with the center of section. ${ }^{(2)(8)}$ In the container ship, however, the shear center in way of hatch openings lies under the bottom and so-called coupling lever becomes large. Though the center of torsion does not necessarily agree to the shear center and is subjected to the effect of the variable section in the actual ship, the qualitative discussions on the shear center are made.

The position of the shear center on the open section shown in Fig. 7 is given by the following well known formula. 


$$
e=\frac{h^{2} b^{2} t}{4 I}
$$

The equation (24) is, however, introduced under assumption that the shear deflection of the plates in their plane is neglected. If the shear deflection is taken into consideration, the cross section rotates unticlockwise about the point $S^{\prime}$ shown in Fig. 7 (b). The amount of the translation of the Web plate $\left(\phi e^{\prime}\right)$ due to the rotation equals to the shear deflection of the beam.

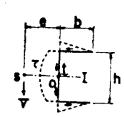

(0)

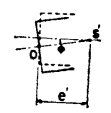

(b)

Fig. 7 Shear center and effect of shear deflection.

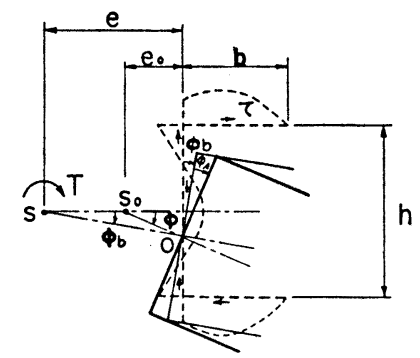

Fig. 8 Virtual center of tersion.

On the other hand, the cross section rotates clockwise about the shear center $S$ shown in Fig. 8 when the beam is subjected to the torsional moment and the shear rigidity is infinity. However the section rotates about the center of Web plate by the shear deflection of the plates in their plane and a virtual center of torsion becomes the point $S_{0}$ shown in Fig. 8. Though the effective shear center may be defined by the combination of two mechanism mentioned above, it is left for the future work.

\section{3 Hydrodynamical inertia, centre of virtual mass.}

In the coupled torsional-horizontal vibration the cross section of ship undergoes both translational and rotational motion, therefore both of the virtual mass and the virtual moment of inertia of the water should be taken into account for determining the vibration characteristics. Generally speaking the hydrodynamical inertia in the compound motion such as a combination of translation and rotation cannot be separated into the parts which are attributed to the individual component motions. Whether the separation is possible or not in the torsional-horizontal vibration should be examined*). Suppose a two-dimensional section is floating on water and is subject to horizontal translation with velocity $U$ and rotational motion about 0 with angular velocity $\omega$ as shown in Fig. 9. The section is assumed to be symmetric about $y$-axis. Provided that the water to be non-viscous and incompressible, the velocity potential $\phi$ for the combined motion of translation and rotation can be expressed by the sum of $\phi_{1}$ and $\phi_{2}$ which are the velocity potentials for translation and rotation respectively. The pressure acting on the surface of the section can be obtained by the

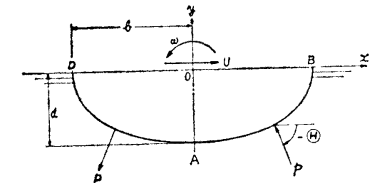

Fig. 9 Coordinate system of a floating body. following formula,

$$
p=\rho\left(\frac{\partial \phi_{1}}{\partial t}+\frac{\partial \phi_{2}}{\partial t}\right)
$$

where $\rho$ is density of water. As $\phi_{1}$ and $\phi_{2}$ are the velocity potentials of individual translation and rotation respectively, they are expressed in terms of $U$ and $\omega$ respectively as follows,

$$
\text { and } \left.\begin{array}{l}
\phi_{1}=\bar{\phi}_{1} U \\
\phi_{2}=\bar{\phi}_{2} \omega
\end{array}\right\}
$$

where $\bar{\phi}_{1}$ and $\bar{\phi}_{2}$ are determined by the section forms and are independent of time. Substituting (26) into (25) the pressure can be expressed by the linear combination of $U$ and $\omega$. Integration of the horizontal component and the moment about 0 (in Fig. 9) of the pressure respectively gives the hydrodynamical force and the moment about 0 respectively, which are given as follws.

$$
\left.\begin{array}{lc}
\text { hydrodynamical force } & F=-F_{1} \frac{d U}{d t}-F_{2} \frac{d \omega}{d t} \\
\text { hydrodynamical moment } & M=-M_{1} \frac{d U}{d t}-M_{2} \frac{d \omega}{d t}
\end{array}\right\}
$$

* Matsuura has treated the problem in a recent report ${ }^{9)}$. 
where the positive directions of $F$ and $M$ coincide with those of $U$ and $\omega$ and

$$
\left.\begin{array}{rl}
F_{1} & =\rho \int_{D A B} \bar{\phi} \cos \Theta d s, \quad F_{2}=\rho \int_{D A B} \bar{\phi}_{2} \cos \Theta d s \\
M_{1} & =-\rho \int_{D A B} \bar{\phi}_{1}(y \cos \Theta-x \sin \Theta) d s, M_{2}=-\rho \int_{D A B} \bar{\phi}_{2}(y \cos \Theta-x \sin \Theta) d s
\end{array}\right\}
$$

In the formulae above mentioned, $F_{1}$ and $M_{2}$ are the virtual mass and the virtual moment of inertia of water respectively since they, multiplied by the linear and the angular acceleration respectively, give the hydrodynamical inertia force and moment respectively. It is to be noted that $F$ has a term including $d \omega / d t$ and $M$ has a term including $d U / d t$. Since $F_{2}$ and $M_{1}$ are not identically zero in general, this means that the horizontal translational motion induces a hydrodynamical couple about 0 and the rotation about 0 induces a hydrodynamical force. Thus in a pure translational motion (rotation is constrained) there acts a hydrodynamical moment about 0 of the magnitude of $-M_{1} d U / d t$. This monent, divided by the hydrodynamic force $F_{1} d U / d t$, gives a linear quantity $y_{G}$ as follows,

$$
y_{G}=-M_{1} / F_{1}
$$

If $y_{G}$ is measured upward from 0 , the point at the end of $y_{G}$ has a meaning analoguous to the centre of mass in a rigid body. This point is called the centre of virtual mass.

In a rigid body dynamics, the moment of inertia about any axis can be expressed in terms of the moment of inertia about centre of mass, the mass and the distance between the axis and the centre of mass by means of the so-called the theorem of parallel axis. If the same relation holds in the case of hydrodynamic inertia, the hydrodynamical inertia in any two-dimensional motion can be determined by the virtual mass, centre of virtual mass and the virtual moment of inertia about any axis perpendicular to the plane of motion. This is to be examined in the present study.

Suppose a two-dimensional body floating on the water shown in Fig. 10 is in motion and the translational and the rotational velocity at a point $K$ are $\dot{x}$ and $\dot{\beta}$ respectively. Then, using the geometrical

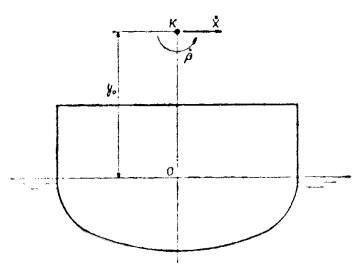

Fig. 10 Illustration of a floating body in plane motion. relations and (27), the force $F_{k}$ and the moment $M_{k}$ acting at $K$ are,

$$
\left.\begin{array}{rl}
F_{k} & =-F_{1} \ddot{x}-\left(F_{1} y_{0}+F_{2}\right) \ddot{\beta} \\
M_{k} & =-\left(M_{1}+F_{1} y_{0}\right) \ddot{x}-\left\{M_{2}+\left(F_{2}+M_{1}\right) y_{0}+F_{1} y_{0}^{2}\right\} \ddot{\beta}
\end{array}\right\}
$$

If the translational velocity $\dot{x}=0$,

where

$$
\begin{aligned}
F_{k} & =-\left(F_{1} y_{0}+F_{2}\right) \ddot{\beta} \\
M_{k} & =-I_{w k} \ddot{\beta}
\end{aligned}
$$

$$
I_{w k}=M_{2}+\left(F_{2}+M_{1}\right) y_{0}+F_{1} y_{0}^{2}
$$

$I_{w k}$ is the total moment of inertia about $K$ arising from the hydrodynamic inertia. (Kumai calculated the $I_{w k}$ and pointed out that the centre of rotation should be taken into consideration for the calculation of $I_{w k}$.)

If the virtual mass and moment of inertia shown in (28) and the centre of virtual mass shown in (29) are treated as if in the rigid body, the total moment of inertia takes the following form.

$$
I_{k}=M_{2}+F_{1}\left(y_{0}-y_{G}\right)^{2}=M_{2}+2 M_{1} y_{0}+F_{1} y_{0}^{2}
$$

$I_{k}$ and $I_{w k}$ are equal if the relation $F_{2}=M_{1}$ holds. It can be shown that this relation exists in a common ship section such as Lewis forms and it can be concluded that the hydrodynamic inertia can be treated as the inertia in a rigid body motion. Thus we can set up the equation of motion of floating body if the virtual mass, virtual moment of inertia about $O$ in Fig. 9 and the position of centre of virtual mass are known. Virtual mass and moment of inertia has already obtained by Kumai11), Landweber ${ }^{15)}$ and 
other authors for practical ship sections. Hence in this report the centre of virtual mass is calculated for Lewis forms. If the st:p section in $z$-plane is the transformed image of the unit circle in $\zeta$-plane by the following function

$$
z=b_{0}\left(\zeta+\sum_{n=1}^{\infty} \frac{a_{n}}{\zeta^{n}}\right)
$$

$F_{1}, F_{2}, M_{1}$ and $M T_{2}$ are in the following forms.

$$
\begin{aligned}
& F_{1}=2 \rho b_{0}{ }^{2} \int_{-\pi / 2}^{0} \Phi_{1}\left\{\left(1-a_{1}\right) \cos \theta-\sum_{n=2}^{\infty} n a_{n} \cos n \theta\right\} d \theta \\
& F_{2}=2 \rho b_{0}^{2} \int_{-\pi / 2}^{0} \Phi_{2}\left\{\left(1-a_{1}\right) \cos \theta-\sum_{n=2}^{\infty} n a_{n} \cos n \theta\right\} d \theta \\
& M_{1}=-2 \rho b_{0}^{2} \int_{-\pi / 2}^{0} \Phi_{1}\left[\left\{\left(1-a_{1}\right) \sin \theta-\sum_{n=2}^{\infty} a_{n} \cos n \theta\right\}\left\{\left(1-a_{1}\right) \cos \theta-\sum_{n=2}^{\infty} n a_{n} \cos n \theta\right\}\right. \\
&\left.-\left\{\left(1+a_{1}\right) \cos \theta+\sum_{n=2}^{\infty} a_{n} \cos n \theta\right\}\left\{\left(1+a_{1}\right) \sin \theta+\sum_{n=2}^{\infty} n a_{n} \sin n \theta\right\}\right] d \theta \\
& M_{2}=-2 \rho b_{0}^{2} \int_{-\pi / 2}^{0} \Phi_{2}\left[\left\{\left(1-a_{1}\right) \sin \theta-\sum_{n=2}^{\infty} a_{n} \sin n \theta\right\}\left\{\left(1-a_{1}\right) \cos \theta-\sum_{n=2}^{\infty} n a_{n} \cos n \theta\right\}\right. \\
&\left.\left.-\left\{\left(1+a_{1}\right) \cos \theta+\sum_{n=2}^{\infty} a_{n} \cos n \theta\right\}\left\{\left(1+a_{1}\right) \sin \theta+\sum_{n=2}^{\infty} n a_{n} \sin n \theta\right\}\right] d \theta\right\}
\end{aligned}
$$

In the Lewis forms the coefficients in (33) are as follows.

$$
\begin{aligned}
& b_{0}=\frac{b}{4}\{3(1+\lambda) \\
& \left.-\sqrt{ }(1+\lambda)^{2}+8 \lambda(1-4 \sigma / \pi)\right\} \\
& a_{1}=(1-\lambda) b /\left(2 b_{0}\right) \\
& a_{3}=(1+\lambda) b /\left(2 b_{0}\right)-1
\end{aligned}
$$

where

$$
\lambda=d / b \text { and } \sigma=S / 2 b d
$$

( $S$ : sectional area)

The centre of virtual mass is given by (29).

This formula has been calculated for several cases and the results are shown in Fig. 11*). It is found from this figure that the centre of virtual mass is located at about halfway of draught from free surface when $\lambda=$ 5.0. The centre becomes higher with decreasing $\lambda$. This means that the section having small breadth and large draught has the centre of virtual mass at the lower position and vice versa. In a very flat body the centre of mass goes above free surface.

* Yamakoshi also calculated the centre of virtual mass ${ }^{16)}$ using the results of Kumai ${ }^{10)}$. The results agree with Fig. 11.

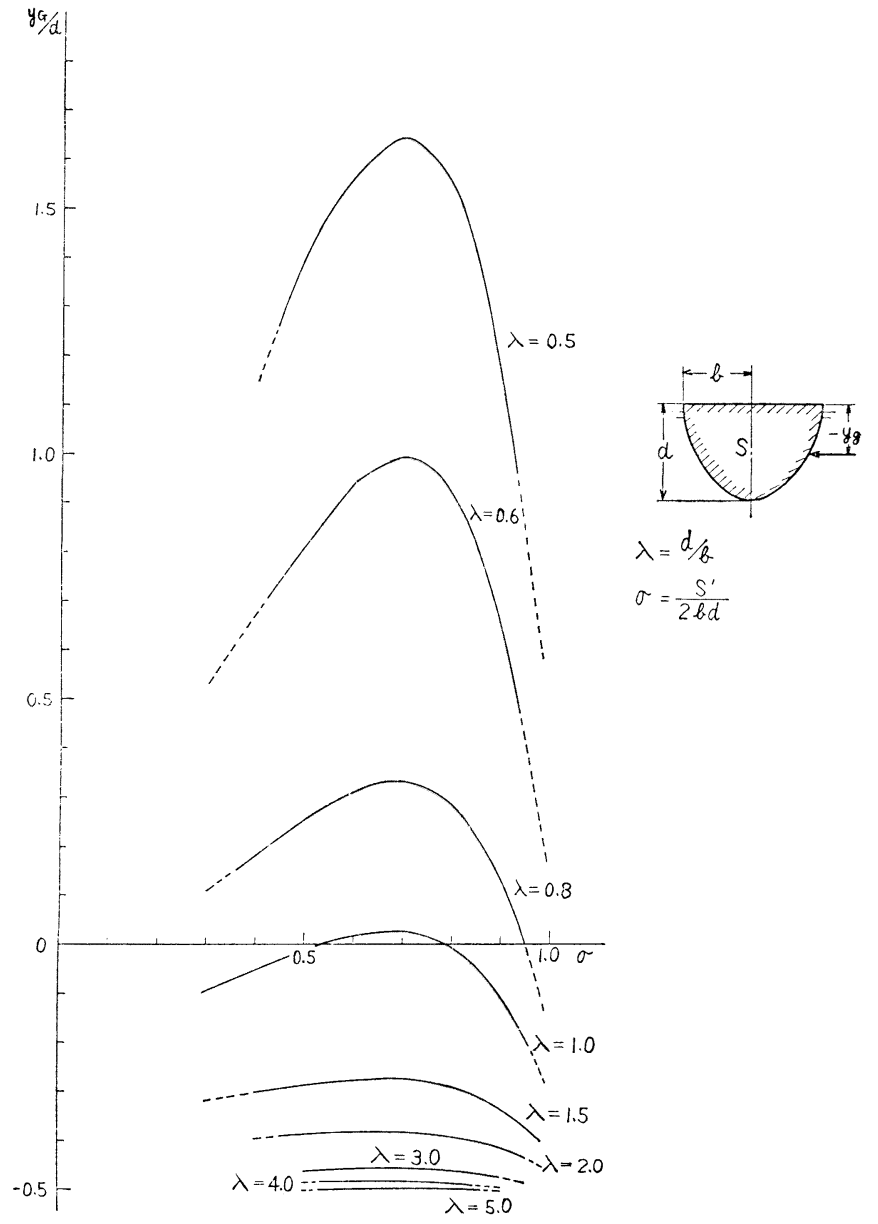

Fig. 11 Position of centre of virtual mass of the Lewis forms. 

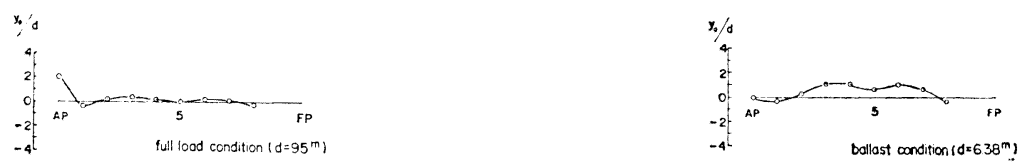

Fig. 12 Centre of virtual mass of the container ship.

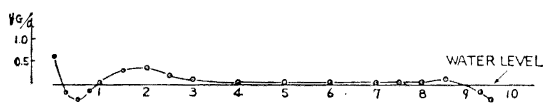

Fig. 13 Centre of virtual mass of a 83,000 ton tanker.

The centre of the virtual mass in actual ships is shown in Fig. 12 and Fig. 13 . The former is the centre for the container ship studied in the present report. The latter is for a tanker of 83,000 tons D.W.. It is found from the figures that the centre of the virtual mass is about the free surface in the full load condition and it is above the free surface by the height of nearly the draught in the ballast condition**).

\section{Model Experiments}

\subsection{Procedure of experiments}

Alluminium models have been used for the vibration experiments which were originally made for the investigation of the statical strength of the container ship (14). The models used are the following two types; ship-shaped beam (Model A) and box beam (Model D). Their principal particulars are shown in Table 3, the details of the models are seen in the paper mentioned above(14). Models are hung with even keel and excited at one end horizontally. Fig. 14

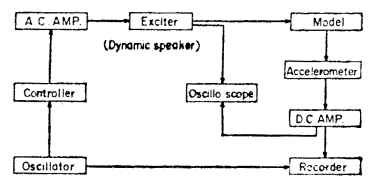

Fig. 14 Block diagram of model experiments. shows the block diagram of the experiments.

Table 3 Principal particulars of alluminium models

\begin{tabular}{|c|c|c|c|c|c|}
\hline Model & $L \times B \times D$ & weight & ship side & bottom & ship form \\
\hline $\begin{array}{l}A \\
D\end{array}$ & $\begin{array}{c}\mathrm{mm} \mathrm{mm} \mathrm{mm} \\
1968 \times 325 \times 194 \\
\text { Do. }\end{array}$ & $25.5^{\mathrm{kg}}$ & $\begin{array}{c}\text { partial double hull } \\
\text { single hull }\end{array}$ & $\begin{array}{l}\text { double } \\
\text { Do. }\end{array}$ & $\begin{array}{c}\text { ship-shaped beam } \\
\text { box beam }\end{array}$ \\
\hline
\end{tabular}

\subsection{Results discussions}

Measured mode curves and frequencies are shown Fig. 15, Fig. 16 and Table 4. For the comparisor. the calculation of the vibration has been carried out under the following assumptions.

(a) Though the effect of the warping exist in the ships with large opening in decks, it is difficul1 to evaluate quantitatively the warping rigidity for the complex structure. So the reduced thickness method $^{(8)}$ has been applied and the Saint-Venant torsional rigidity is used.

(b) The center of torsion is assumed to lie along the keel line over the length of the beam.

(c) The distance between the center of torsion and the center of gravity on the midship sectior is used as coupling lever and assumed constant over the length.

** In the previous study of one of the authors it was shown that the centre of virtual mass is nearly the half draught from bottom ${ }^{13)}$. In that study the moment of the vertical component of thi pressure was ignored, which is, from Fig. 11 valid only the slender section. For general shape: the conclusion in the present report should be preferable. 

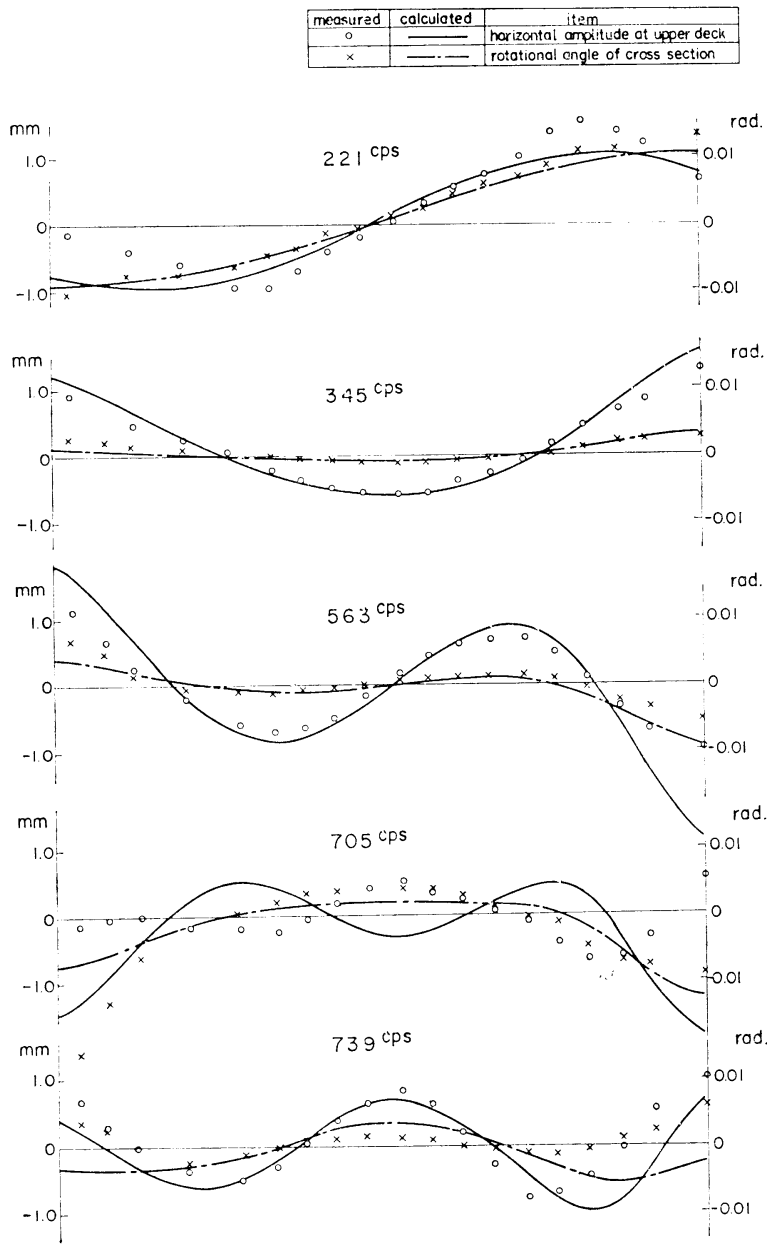

Fig. 15 Comparison between measured and calculated mode curves of models tested.
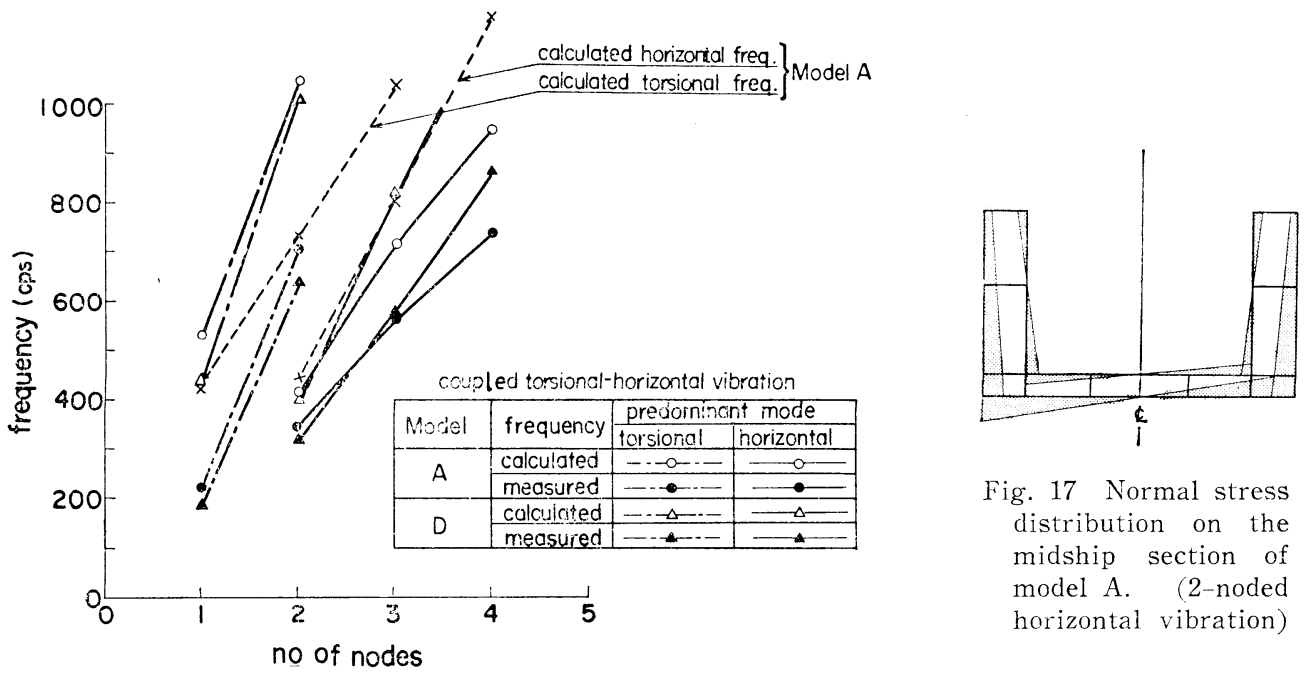

Fig. 17 Normal stress distribution on the midship section of model A. (2-noded horizontal vibration)

Fig. 16 Relation between frequency and number of nodes of models tested. 
Table 4 comparison between measured and calculated frequencies of models

\begin{tabular}{|c|c|c|c|c|c|c|c|c|c|}
\hline \multirow{3}{*}{ Model } & \multicolumn{3}{|c|}{ measured } & \multicolumn{3}{|c|}{ calculated } & \multirow{3}{*}{$\begin{array}{l}\text { modified } \\
\text { frec. cps }\end{array}$} & \multirow{3}{*}{ (A) $/$ B } & \multirow{3}{*}{ (A) $/ C$} \\
\hline & \multicolumn{2}{|c|}{ No. of nodes } & \multirow{2}{*}{ freq. cps } & \multicolumn{2}{|c|}{ No. of nodes } & \multirow{2}{*}{ freq. cps } & & & \\
\hline & horizontal & torsional & & horizontal & torsional & & & & \\
\hline \multirow{5}{*}{ A } & 1 & (1) & 221 & 1 & (1) & 536.5 & 221 & 0.412 & 1. 000 \\
\hline & (2) & 2 & 345 & (2) & 2 & 412.6 & 345 & 0.836 & 1.000 \\
\hline & (3) & 3 & 563 & (3) & 3 & 715.0 & 598 & 0.787 & 0.942 \\
\hline & 3 & (2) & 705 & 4 & (2) & 1042.4 & 429 & 0.676 & 1. 613 \\
\hline & (4) & 4 & 739 & (4) & 2 & 948.6 & 793 & 0.779 & 0.931 \\
\hline \multirow{5}{*}{$\mathrm{D}$} & 1 & (1) & 186 & 1 & (1) & 453.2 & 186 & 0.410 & 1.000 \\
\hline & (2) & 2 & 320 & (2) & 2 & 401.4 & 320 & 0.797 & 1.000 \\
\hline & (3) & 3 & 576 & (3) & 3 & 823.4 & 656 & 0.700 & 0.878 \\
\hline & 3 & (2) & 638 & 2 & (2) & 1011.7 & 415 & 0.631 & 1.537 \\
\hline & (4) & 4 & 866 & & & & & & \\
\hline
\end{tabular}

note) (1), (2) $\cdots \cdot$ mean the predominant modes.

(d) The calculation method is the numerical computation method developed by one of the authors in the previous paper(2).

The results of the calculation are shown in Fig. 15, 16 and Table 4 together with the measured ones. From these we see the followings.

(1) Measured frequencies are pretty lower than the calculated ores. The reason of this is supposed to be the reduction of rigidities in models due to the imperfect stress flow into inner huil. To check this in more detail, the vibratory normal (bending) stress at the resonance of 2 -noded vibration has been measured and the results are shown in Fig. 17. It can be seen from this figure that the stress in the inner hull is considerable lower than that on the outer hull. The models have riveted constructions and sometimes during the experiment some riveted joints were loosened. As the actual ship is built by welded construction, such a defect may not happen.

(2) If the calculated frequencies are modified so as to make the lowest calculated frequency coincide with the measured one and the same ratio is multiplied to the higher frequencies, the higher frequencies calculated also coincide with the measured ones, as shown in Table 4, with the exception of two-noded torsional vibration which is subjected to significant coupling effect.

(3) Measured and calculated mode curves show good agreement except for the horizontal amplitude at $705 \mathrm{cpm}$ (two-noded torsion predominant mode).

From the observations mentioned above, it is concluded that the assumptions used in the calculation are appropriate with the exception for the rigidities mentioned above. For the detailed ciscussion of th: actual ship structure, however, it is necessary to introduce the practical method for computatinn, taking into account of the warping rigidity including the shear effect.

\section{Experiments in Fuil Scale Ship}

\subsection{Procedure of experiments and the results}

The ship tested is a 15,800 tons D. W. container ship of length $175 \mathrm{~m}$ (between perpendiculars) whose particulars are shown in Table 5. Two kinds of tests have been carried out, $i . e$. the experiment by means of the vibration generator in port and the running test in sea trial. In the test using the vibration exciter (simply called as "exciter test" hereafter) direction of the exciting force has been set both in vertical and horizontal. In any case of exciter test and sea trial both the vertical and the transverse vibration have been measured (measuring points are shown in the mode 
curves). The test condition is shown in Table 5. The results are stated in the following part.

Table 5 Particulars of the Ship and the test condition.

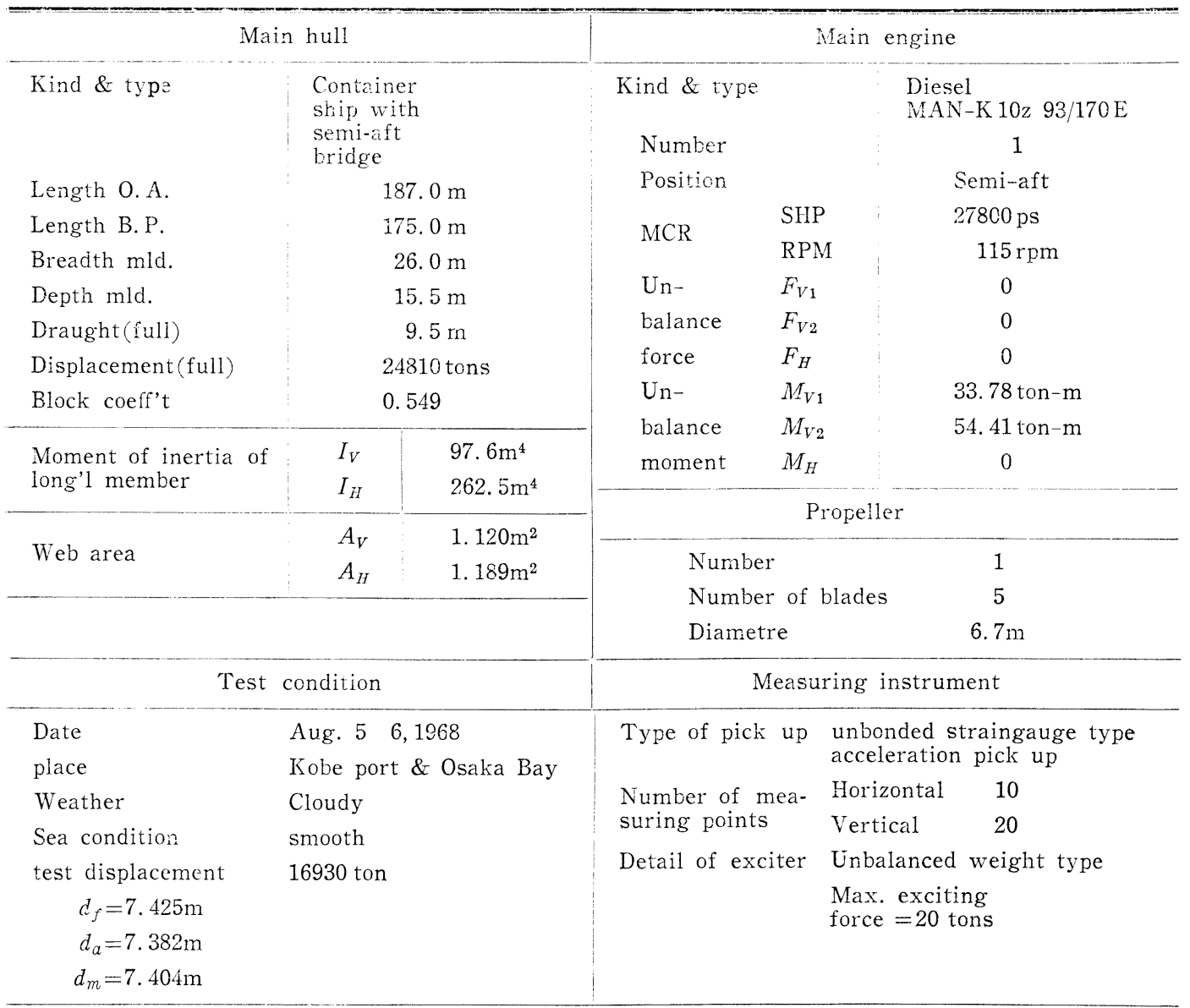

\section{Vertical vibration}

Vertical vibration has been measured at 18 locations on both sides of the upper deck and 2 locations on the double bottom in the container hold. The resonance curves at the major measuring points in exciter test are shown in Fig. 18. The mode curves at the resonant peaks are shown in Fig. 20, from which the resonant frequency, number of nodes and the acceleration per unit exciting force are listed in Table 6. From these figures and tables the following observation is made.

(a) By this exciter test the resonant frequency at different measuring points coincide up to 8 nodes and the corresponding measured mode curves show the beam vibration behaviour. But in the higher modes the wave length of the mode curves (distance between neighbouring nodal position) becomes irregular and the amplitudes at ends become large compared with those at midship.

(b) As is seen in Fig. 18, there is no prominent peak in the range above 3 nodes. In this range the phenomenon of the stern vibration is observed and the mode curves become very complex. Thus the behaviour based on the beam theory is seen up to 8 noded mode in this ship.

As there are many kinds of exciting forces in the sea trial, the recorded wave profile of the vibration is very complex as shown in Fig. 24. Thus the Fourier analysis of the waves forms has been made by means of the data processor consists of the digital computer, AD- and DA-converter and the input/output system instalied in the Nagasaki Technical Institute. The resonance curves 

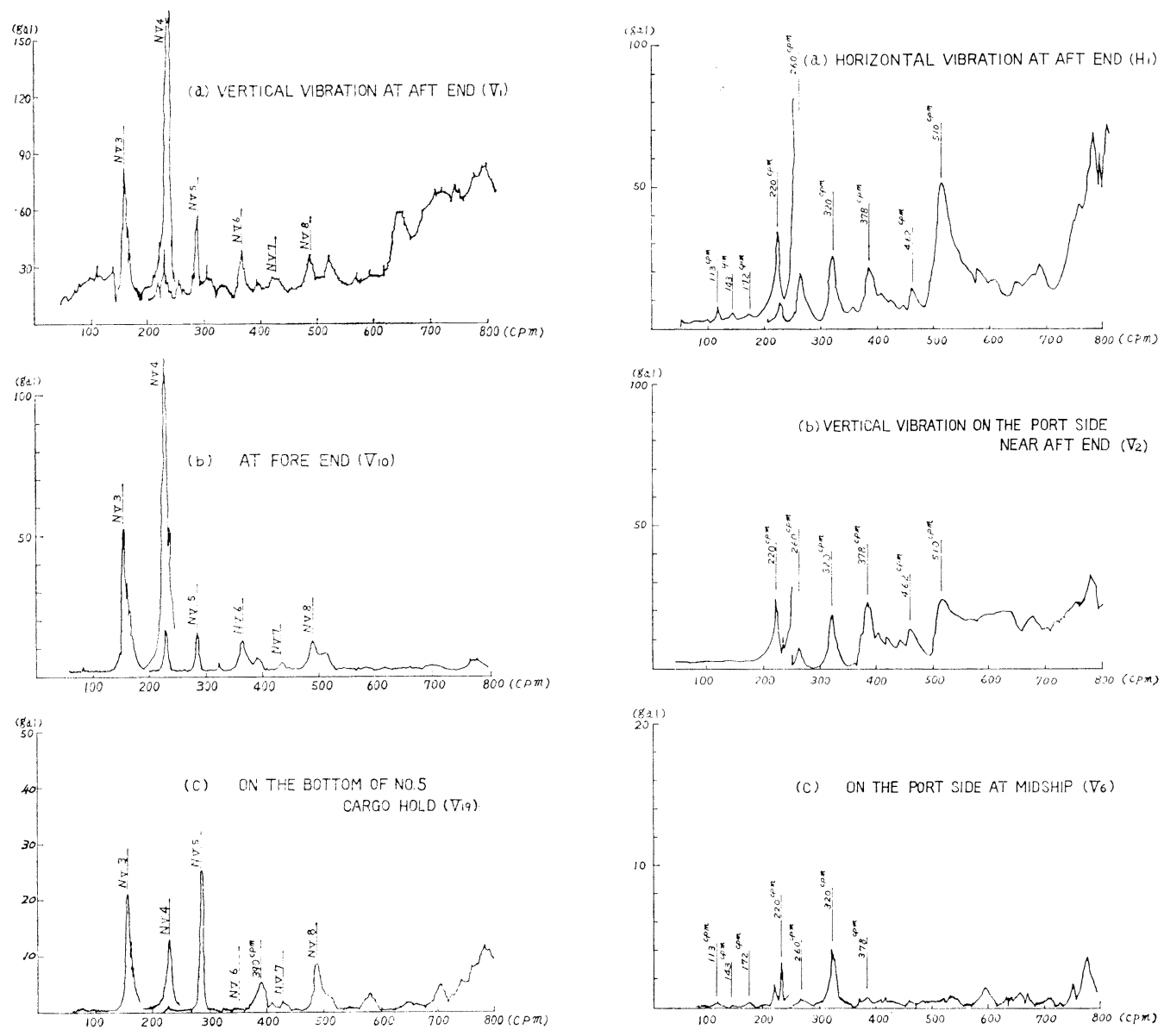

Fig. 18 Resonance curves at the major measuring points in vertical excitation of exciter test.

Fig. 19 Resonance curves at the major measuring points in horizantal excitation of exciter test.

Table 6 Resonant frequencies and amplitudes of hull vertical vibration

\begin{tabular}{|c|c|c|c|c|c|c|c|}
\hline \multicolumn{3}{|c|}{ Exciter test (gal/ton) } & \multicolumn{4}{|c|}{ Sea trial (gal) } & \multirow{2}{*}{ No. of nodes } \\
\hline freq & $\begin{array}{c}\text { aft end } \\
\left(V_{1}\right)\end{array}$ & $\begin{array}{l}\text { fore end } \\
\left(V_{10}\right)\end{array}$ & freq & $\begin{array}{c}\text { aft end } \\
\left(V_{1}\right)\end{array}$ & $\begin{array}{l}\text { fore end } \\
\left(V_{10}\right)\end{array}$ & $\begin{array}{l}\text { multiples } \\
\text { of rpm. }\end{array}$ & \\
\hline \multicolumn{2}{|c|}{$\mathrm{cpm}$} & & & 15.0 & 15.0 & $\mathrm{rpm} \times 1$ & 2 \\
\hline 154 & 32.5 & 21.2 & 152 & 17.0 & & 2 & 3 \\
\hline 227 & $\begin{array}{l}28.0 \\
65.0\end{array}$ & $\begin{array}{l}19.0 \\
28.4\end{array}$ & 222 & 32.0 & 15.0 & 2 & 4 \\
\hline 284 & 62.0 & 18.3 & 290 & 12.0 & & 5 & 5 \\
\hline 367 & 26.0 & 8.7 & 370 & 16.0 & 4.0 & 5 & 6 \\
\hline 431 & 11.4 & 2.4 & 432 & 22.0 & 10.5 & 4 & 7 \\
\hline 490 & 13.3 & 4.8 & & & & & 8 \\
\hline
\end{tabular}

and the mode curves thus obtained are shown in Fig. 22 and Fig. 24. From these figures it is seen that

(c) Up to 7 -noded mode can be perceived. The resonance frequencies, as shown in Table 6, agree with those obtained by exciter test.

(d) In the vicinity of the resonant frequency of 6-noded vibration there exists the resonant peak 

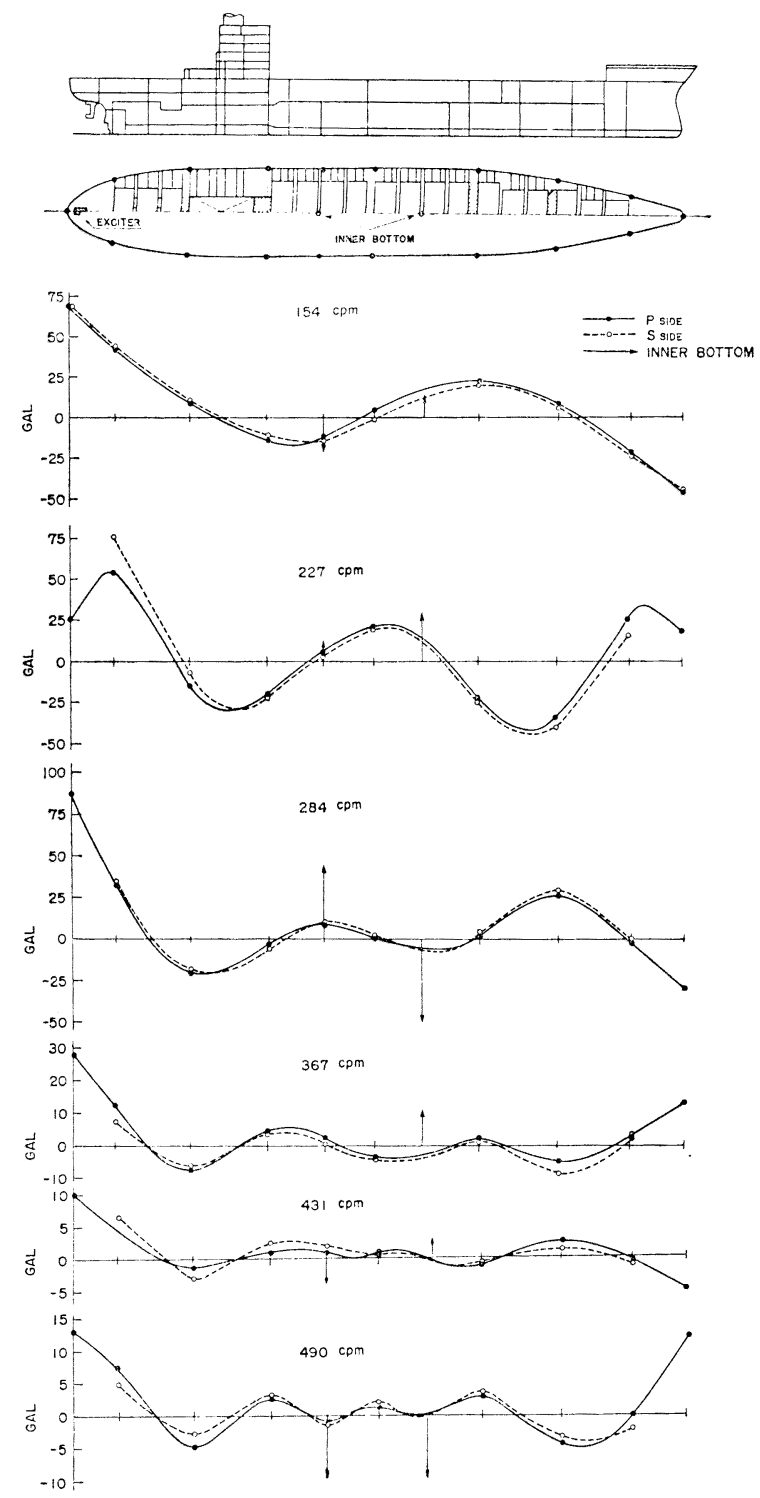

Fig. 20 Mode curves in vertical excitation of exciter test.

of the torsion-horizontal vibration. Therefore the amplitudes on both sides do not coincide with, because of the interference of the torsional-horizontal vibration.

\section{Torsion-Horizontal vibration}

As we have no pick-ups for angular amplitude, the torsional vibration is measured as a vertical amplitucies and the phase differences at the both sides of upper deck. The measured resonance curves and the mode curves are shown in Fig. 19 and Fig. 21. From the figures the following are observed.

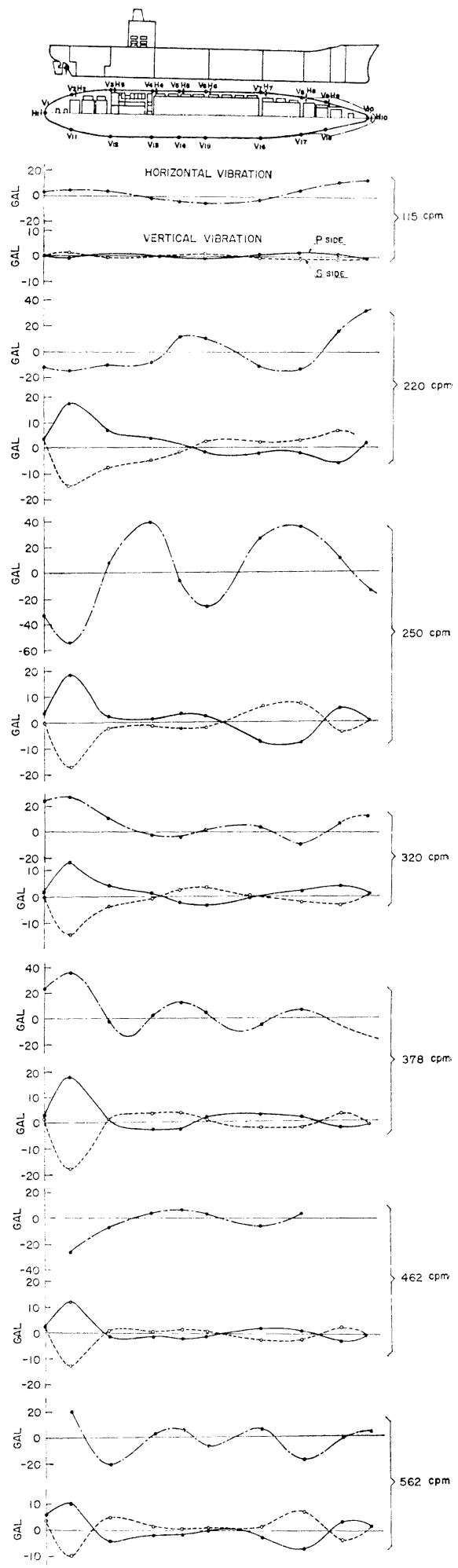

Fig. 21 Mode curves in horizontal excitation of exciter test. 

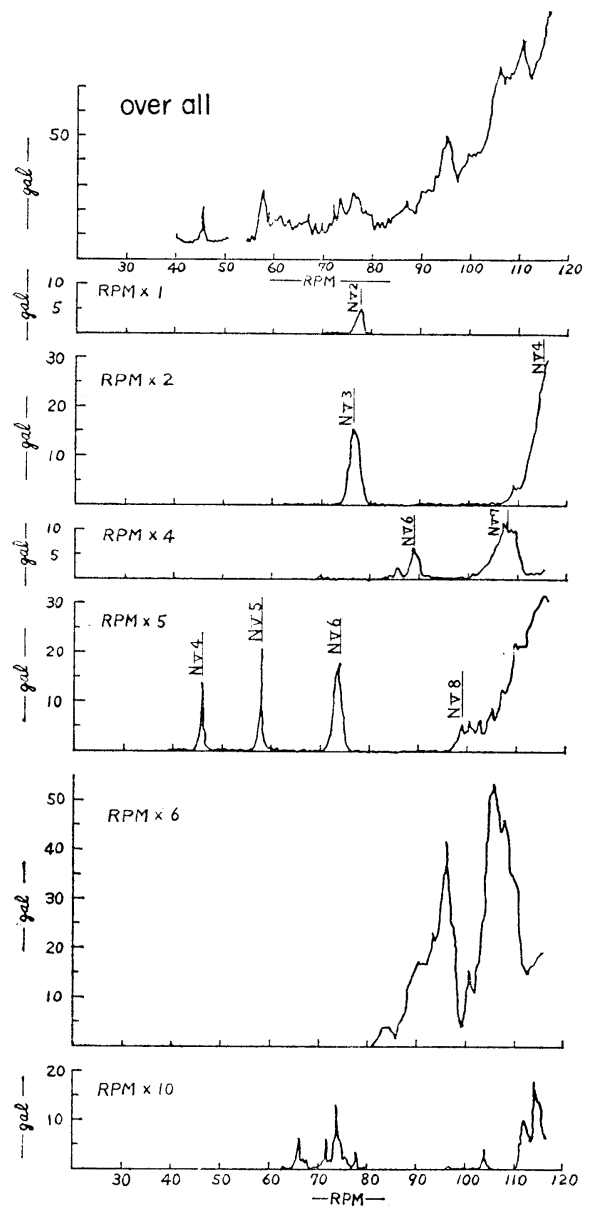

Fig. 22 Resonance curves of vertical
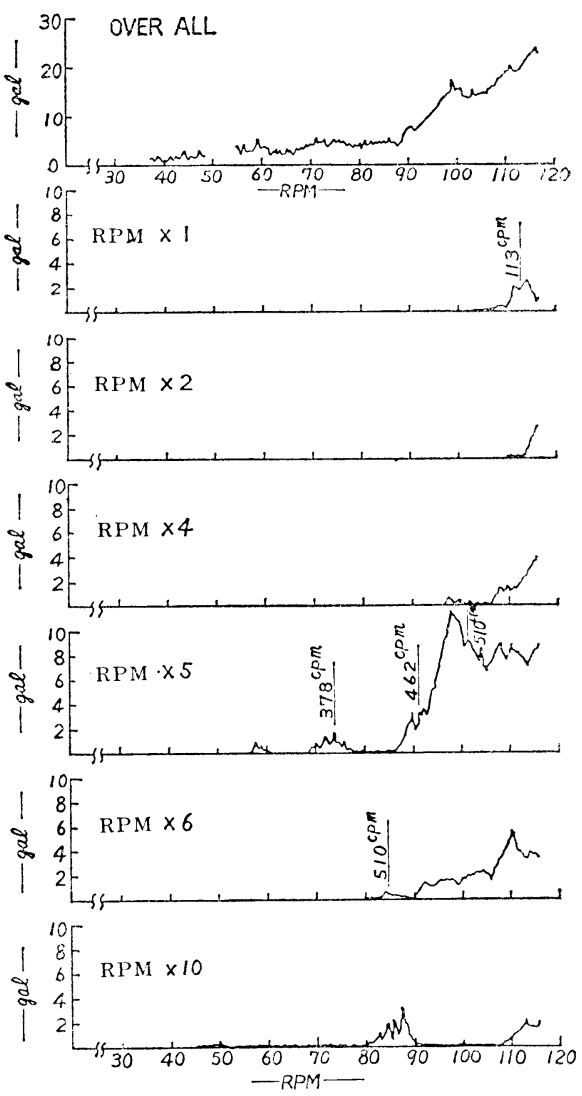

Fig. 23 Resonance curves of horizontal vibration at aft end $(H)$.

Table 7 Resonant frequencies and amplitudes of horizontal and torsional vibration.

\begin{tabular}{|c|c|c|c|c|c|c|c|c|}
\hline \multirow{3}{*}{ Mode } & \multicolumn{5}{|c|}{ Measured } & \multicolumn{3}{|c|}{ Calculated } \\
\hline & \multirow{2}{*}{ Frequency } & \multicolumn{2}{|c|}{ No. of nodes } & \multicolumn{2}{|c|}{ Acceleration (gal/ton) } & \multirow{2}{*}{ Frequency } & \multicolumn{2}{|c|}{ No. of nodes } \\
\hline & & Horizontal & Torsional & $\begin{array}{l}\text { Aft end }\left(\mathrm{H}_{1}\right) \\
\text { Horizontal }\end{array}$ & $\begin{array}{l}\text { Near Aft end } \\
\left(V_{2}\right) \text { Vertical }\end{array}$ & & Horizontal & Torsional \\
\hline 1 & 113 & 2 & 3 & 11.4 & - & 90.6 & 2 & 2 \\
\hline 2 & 143 & - & - & 6.0 & - & 135.3 & 1 & 1 \\
\hline 3 & 172 & - & 一 & 9.0 & - & 175.9 & 3 & 2 \\
\hline 4 & 220 & 3 & $3(1)$ & 12.8 & 9.4 & 190.2 & 2 & 2 \\
\hline 5 & 260 & 4 & 4 & 26.7 & 9.4 & 257.3 & 4 & 4 \\
\hline 6 & 320 & 4 & 2 & 23.6 & 16.5 & 301.3 & 3 & 3 \\
\hline 7 & 378 & 5 & 3 & 13.7 & 15.0 & 343.0 & 5 & 5 \\
\hline 8 & 462 & (3) & $5(3)$ & 6.3 & 3.8 & 382.0 & 4 & 4 \\
\hline 9 & 510 & 6 & 6 & 17.9 & 8.6 & 429.8 & 6 & 6 \\
\hline 10 & - & - & - & - & - & 463.1 & 7 & 5 \\
\hline
\end{tabular}




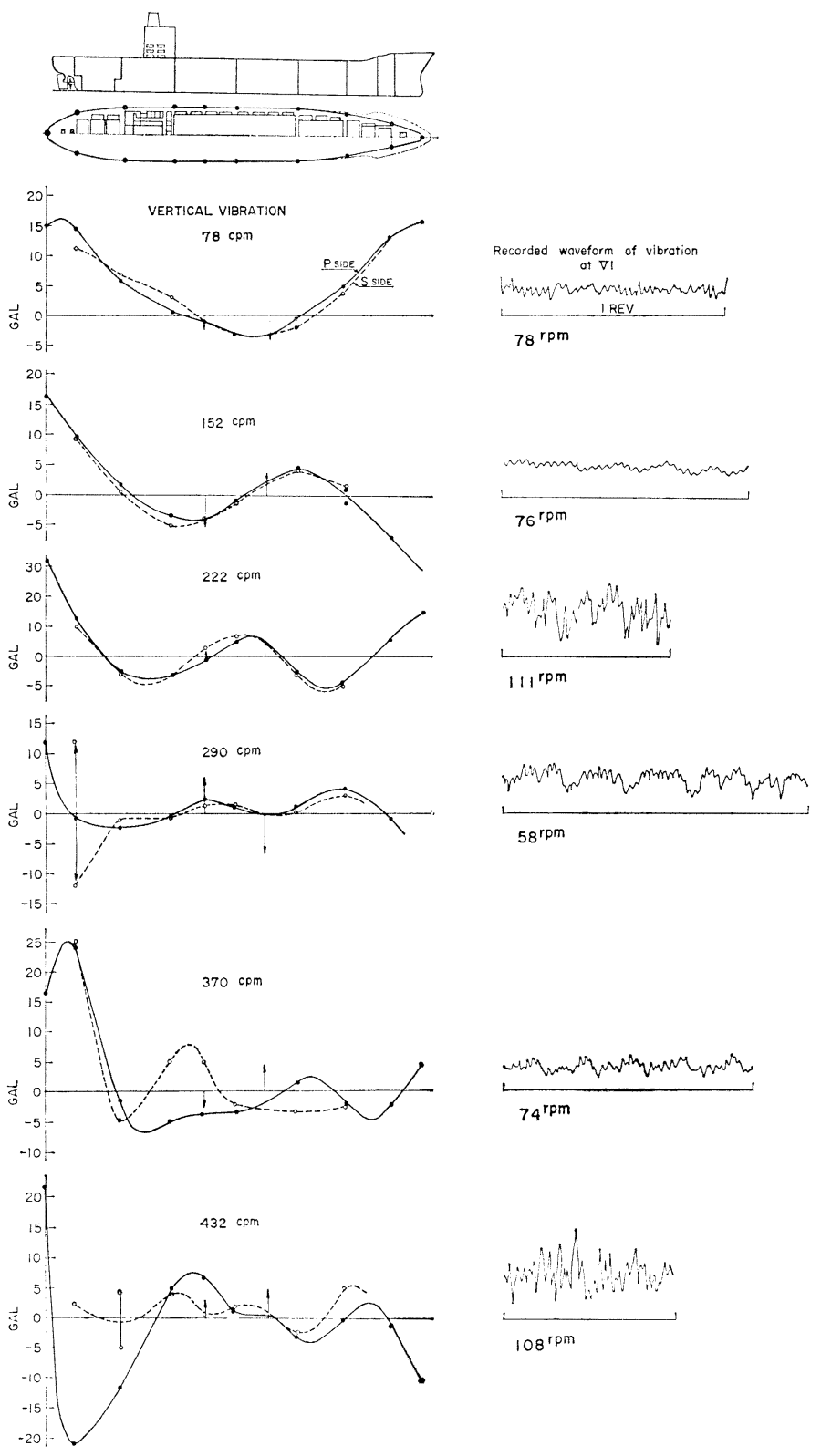

Fig. 24 Mode curves of vertical vibration in sea trial.

(a) In the horizontal and the torsional vibration the horizontal and the torsional amplitudes coexist and their shapes are complex. Hence the classification by the number of nodes can not so easily be made as in the vertical vibration. For convenience's sake, however, the measured modes are classified by the number of nodes and are shown in Table 7. In the determination of the number of nodes in torsional vibration, the observed amplitude at the deck side is divided by the half breadth of the deck at that section. In some cases the mode does not cross the zero line. In such cases the number of nodes are counted by the number of the inflection points of the mode curves.

(b) The mode shapes are very complex but the vertical amplitudes at the both side are nearly 
equal and in opposite direction. Thus the torsional modes have been observed.

In the sea trial the data are complicated and the Fourier analysis mentioned in the vertical vibration has been made and the resonance curves and the mode curves thus obtained are shown in Fig. 23 and Fig. 25. It is seen from these figures that,

(c) In the horizontal vibration the shapes of the resonant peaks are not so sharp as in the vertical vibration. No prominent peak of torsional vibration has been observed. As the measured mode shapes are complex, the number of nodes is difficult to be counted. In the classification in Table 7 , the measured modes are classified compared with the frequency of the exciter test.

\section{2 Discussion of vertical vibration}

The relation between the frequency and the number of nodes are shown in Fig. 26. In the figure the calculated frequency by the digital computer is also shown. The calculation is based on the method in the previous report of one of the authors ${ }^{(6)}$. In the calculation $A$ the shear rigidity is calculated by the energy method(6) and in the calculation $B$ the Web area method is used. At a first glance the calculation A gives the results in closer agreement with the measured results. A closer inspection of the results, however, lead us to the conclusion that the calculation A gives
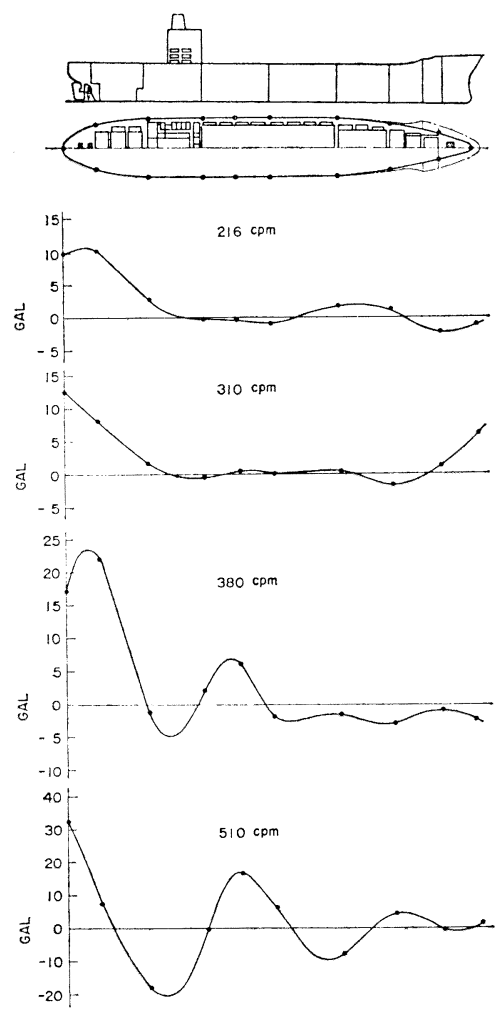

Fig. 25 Mode curves of horizontal vibration in sea trial. underestimated frequencies in the lower modes and agree with the measurements in the higher modes and that the calculation $B$ gives the results in closer agreement with the measured values than calculation $A$ and overestimates in the higher modes. As the calculation is based on the beam theory, it should explain the behaviours in the lower modes. In view of this and the discussion given in $1 \sim 3$, the calculation $B$ is considered to be preferable. The reason of discrepancy in the higher modes is attributed to the non-beam behaviour, which in this ship is the coupling effect with bottom vibration. One of the

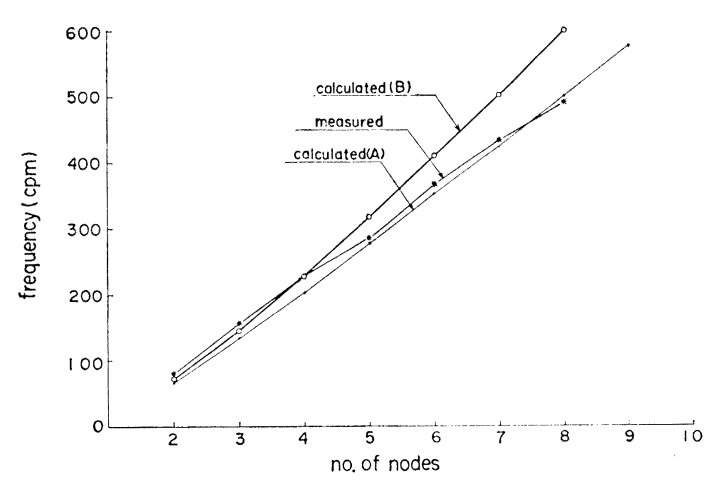

Fig. 26 Relation between frequencies and number of nodes of vertical vibration. authors studies the coupled vibration of main hull with bottom panels(18), the results of which shows that the frequency is considerably lowered by the coupling effects. As an experimental verification of the fact that bottom vibration is induced in the higher modes, the measured vibration on the bottom is large above the 5 -noded modes as shown in Fig. 20.

\section{3 Discussion on the torsional-horizontal vibration.}

As the measured results are so complicated that they cannot be easily classified. As a comparison. the calculation has been made under the following assumptions. 
(a) The general procedure is the same as in the calculation of the models mentioned in 2-2. The reduced thickness is used for torsional rigidity and the Saint-Venant rigidity only is taken into consideration.

(b) As the centre of torsion is difficult to be estimated precisely two kinds of position of the centre of torsion are assumed, on the center of section and on the keel line. The results of the calculation have shown that the latter assumption is not realistic since the torsional frequencies becomes quite low. Thus the calculation with the former assumption is presented here.

(c) In view of the good agreement between the calculated and the measured modes of the models, the assumption may be valid that the lever of the coupling (the distance between centre of torsion and the centre of mass) takes the same value along the length as that at midship.

Thus in the calculation this assumption is adopted. The input data for calculation are shown in Fig. 28 and Fig. 29. The results of the calculation are shown in Fig. 27. In Fig. 27 the data are classified

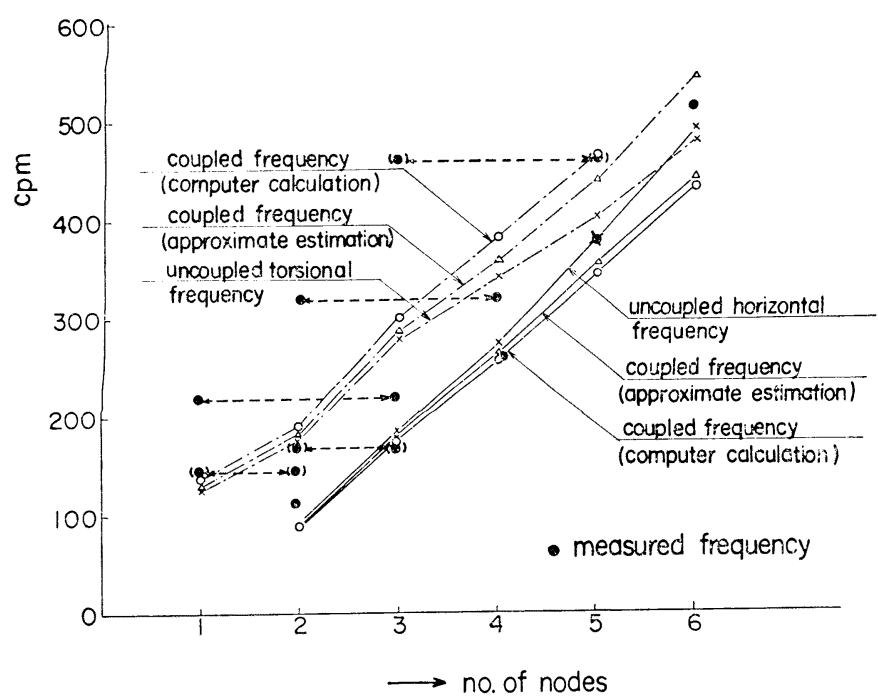

Fig. 27 Relation between frequency and number of nodes (of horizontal mode on upper deck) for horizontal vibration.

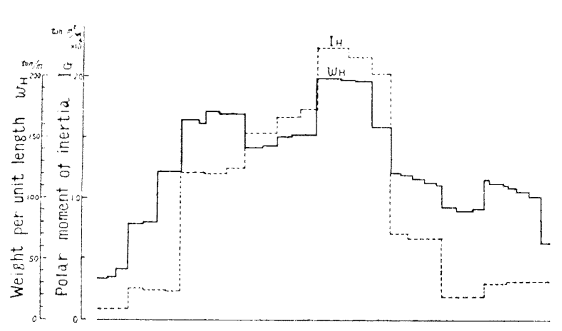

Fig. 28 Weight and polar moment of inertia distributions of the container ship.

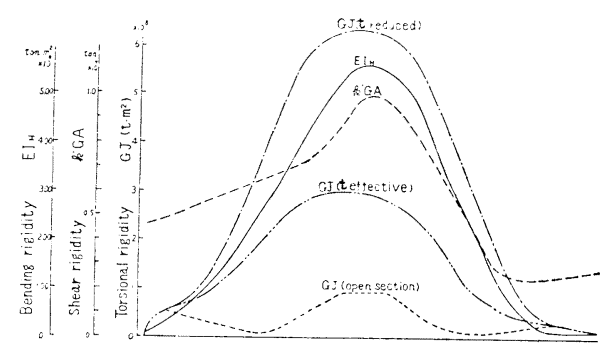

Fig. 29 Bending, shear and torsional rigidity distributions of the container ship.

by the number of nodes of the horizontal mode excepting the uncoupled torsional frequencies for whicli the number of nodes of the torsional mode is used. For comparison the calculated uncoupled horizontal and the torsional frequencies are shown in the figure. It is seen that the frequencies of the torsional vibration go up and those of the horizontal vibration go down ciue to the coupling effect, the former and the latter forming the upper and the lower branch*.) Next the corresponding of the measured and the

*) That such branches exist in the coupled torsional-horizontal vibration was pointed out in the previous report(2). 
calculated frequencies are considered. In each natural frequency the calculated and the measured modes are compared and the measured frequencies are classified in the same manner as the calculated ones. The results are shown in Fig. 27. From this figure it is found that the correspondence of measured and calculated natural frequency is clear in the lower branch while in the upper branch the correspondence is quite poor. (In the measurement the modes at 143 and $172 \mathrm{cpm}$ cannot be obtained since the vibration is so small. At $462 \mathrm{cpm}$ the mode curves are) obtained but imperfect as shown in Fig. 21, so the frequencies of these three modes are set in parenthesis in Fig. 27. The reason why the correspondence between calculated and measured frequency is poor is not known at present. The possible explanation is as follows.

In the present calculation the upper branch is the modification of the torsional mode since the uncoupled torsional frequency is higher than the uncoupled horizontal frequency for identical number of nodes. In the calculation the Saint-Venant torsional rigidity only is used which leads to the possibility of some errors in calculation. The other reason is the assumption of coupling lever. The assumptions that the coupling lever is constant along the length and that the centre of torsion lies at the centre of section may lead to the error in the coupling effect. As is shown in 1-1, higher branch of the coupling is more affected by the coupling. Therefore the effect of error in the coupling calculation is greater in the higher branch than in the lower branch.

At the last of this section the response of the torsionhorizontal vibration is mentioned. As it is difficult to calculate the response of the coupled vibration, the method proposed by A.J. Johnson ${ }^{17)}$ is used. Since his method was developed for the uncoupled vertical and horizontal vibrations, the measured response in exciter test are assumed to be uncoupled horizontal vibration and the dynamic magnification factor has been calculated and compared with those obtained by Johnson. As shown in Fig: 30 the magnification factor of the container ship is not so much different from the conventional type of ship.

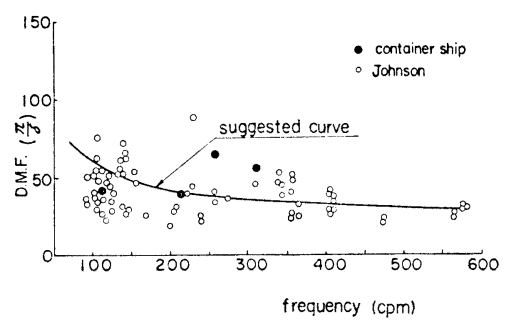

Fig. 30 Dynamic magnification factor of horizontal vibration of container ship compared with that given by Johnson.

\section{Conclusions}

The vertical and coupled torsion-horizontal vibration has been studied on the container ship with theory and experiment. The major conclusions are as follows.

(a) The coupled frequency can be determined by the uncoupled frequencies and the corresponding modes. In the case of the uniform beam the coupled frequency can be obtained by a very simple formula.

(b) Centre of virtual mass for the horizontal vibration of Lewis forms are obtained and it has been found that this centre lies near free surface in the ordinary type of ships in full load condition.

(c) In the actual container ship and model the coupled torsion-horizontal vibration is very complicated and it is not easy to classify the successive modes in it.

(d) The vertical vibration of the container ship is not so much different from that of the conventional type of ship. In the higher modes the effect of the coupling with bottom vibration is observed which should be studied in the future.

(e) As is seen from the discussion throughout the paper, there are many problems remain unknown. Among which the major subjects are the mechanism of the coupled vibration in a complex structure such as ships, the estimation of the rigidity in torsional vibration, the determination of the centre of torsion and the three-dimensional correction factor for virtual mass and virtual moment of inertia of 
water in a very fine ships.

The authors express hearty thanks to Mr. K. Terada for his friendly cooperation in the measurement and data processing. They are also grateful to Mr.T.Hara who extended earnest help to us in making complicated calculations and in preparing the report. Mr.M.Onoue, Mr.M. Matslio and Mr. N. Imamura conducted the difficult measurements with a sincere effort to which the author's acknowledgenents are dedicated.

\section{References}

(1) Kumai, T., "On the Coupled Torsional-Horizontal Vibration of Ships”. J.S.N.A. Japan, Vol. 100 (1957)

(2) Ohtaka, K. et al, "On the Coupled Torsional-Horizontal Vibration of Ships". Mitsubishi Technical Bulletin, No. 54 (1967)

(3) Matsuura, Y., Kawakami, H. and Onogi, H., "Study on the Coupled Torsional and Flexural Vibration of Ships with Large Hatch Openings (2 nd Report)-Coupled Torsional and Flexural Vibration of a Uniform Bar with Open Cross Section-” J.S.N.A. Kansai, Japan, No. 127 (1968).

(4) Yamakoshi, M. and Maeda, Y., "A Consideration on a Coupled Vibration of Horizontal and Torsional Vibration of Ships.” J.S.N.A. West Japan, No. 36 (1968).

(5) Bishop, R.E.D. and Johnson, D.C., "Vibration Analysis Tables". Cambridge Univ. Press (1956).

(6) Ohtaka, K. et al, “A Study of Vertical Vibration of Ships". (1 st and 2 nd report). J. S. N. A. Japan, Vol. 116 (1964) Vol. 119 (1966).

(7) Kumai, T., “Higher Mode Hull Vibration Treated as a Shear Beam”. J. S. N. A. Japan, Vol. 99 (1956).

(8) Kumai, T., "Estimation of Natural Frequencies of Torsional Vibration of Ships". J.S.N.A. West Japan, No. 11 (1956)

(9) Matsuura, Y. and Kawakami, H., "A Consideration on the Added Mass and Added Moment of Inertia of Water in the Coupled Torsional-Horizontal Vibration of Ships”. Vibration Committee Report, J.S.N.A. Japan, V42-9 (1969).

(10) Kumai, T., " $\Lambda$ dded Mass Moment of Water in the Torsional Vibration of Ships". J.S.N.A.Japan, Vol. 104 (1959).

(11) Kumai, T., "Some Corrections on the Added Mass of Water in the Horizontal Vibration of Ships”. J.S.N.A. Japan, Vol. 108 (1960)

(12) Kumai, T., "Three-Dimensional Correction for the Added Mass Moment of Inertia of Water in. the Torsional Vibration of Ships”. J.S.N.A. Japan, Vol. 108 (1960).

(13) Ohtaka, K., Kumai, T. and Lshijima, M., "Study on the Coupled Torsional Horizontal Vibration of Ships. (1 st Rep.)” J.S.N.A. Japan, Vol. 121 (1967).

(14) Mori, M. et al, "On the Torsional Strength of a Ship witi Wide and Long Hatch Openings". J.S.N.A. Japan, Vol. 124 (1968)

(15) Landweber, L. and Macagno, M. C., "Added Mass of Two-Dimensional Forms Oscillating in a Free Surface”. Journal of Ship Research, Vol. 1, (1957)

(16) Yamakoshi, M., "On the Centre of Added Mass of Water in the Coupled Torsional Horizontal Vibration.” Unpublished, (1968).

(17) Johnson, A. J., "On the Amplitudes of Ship's hulls”. Trans. Inst. Engineers \& Shipbuilders in Scotland, (1962).

(18) Ohtaka, K. and Ohyama, T., "Vertical Vibration of Ships Coupled with bottom Vibration. (1 st Report)" In preparation. 